\title{
Synchronous Papillary Thyroid Cancer and Hodgkin's Lymphoma: A Rare Case Report
}

\author{
Lulwah Al Saidan*, Bodour Al Abdulrahim, Khaled Saad Alajmi, Abdulaziz S Almusallam, Mijil Justin and Fawzia \\ Ashkanani
}

ENT, Farwaniya Hospital, Kuwait University, Kuwait

Submission: July 27, 2021; Published: August 11, 2021

*Corresponding author: Lulwah Al Saidan, ENT, Farwaniya Hospital, Kuwait University, Kuwait

\section{Abstract}

Papillary thyroid cancer is the most common thyroid malignancy. Yet it has the best prognosis. The combination of both papillary thyroid cancer and hematological malignancies is considered extremely rare. We present here a case of a young adult with a three-month history of a right neck mass, associated with night sweats and weight loss. A pan CT was done which showed multiple enlarged cervical, axillary and right inguinal lymph nodes. Fine needle aspiration of a thyroid nodule and lymph nodes was done showing a papillary thyroid cancer and high-grade malignant tumor of the lymph nodes. A multidisciplinary team was engaged with proper counseling of the patient was done. He underwent a hemithyroidectomy with an excisional biopsy of the lymph nodes. Histopathology confirmed the diagnosis of papillary thyroid microcarcinoma and Hodgkin's lymphoma. This case delineates the extreme rarity of having synchronous incidence of papillary thyroid cancer and Hodgkin's lymphoma, in addition to the complexity of clinical management due to the limited literature available.

Keywords: Papillary thyroid cancer; Synchronous; Hodgkin’s; Lymphoma; Rare

\section{Introduction}

Papillary thyroid carcinoma is the most prevailing endocrine malignancy, comprises about $80 \%$ of thyroid cancers, with high ratio for lymphatic metastasis, about $10 \%$ of cases present with palpable cervical lymph node metastases [1]. Hodgkin Lymphoma is most common in male gender of 15-27 years old [2], usually presenting with multiple neck lumps, followed by the B symptoms of low-grade fever, night sweat, weight loss. The incidence of synchronous papillary carcinoma and Hodgkin lymphoma is considered very rare. The definite pathophysiology behind it remains not understood. The diagnosis of such cases might be challenging, and there are no standard treatment guidelines.

\section{Case presentation}

A 27-year-old male previously healthy presented to our OPD with three months history of a painless right neck swelling, increasing in size, associated with night sweats, and weight loss of approximately $8 \mathrm{~kg}$. In addition, he also complained of difficulty swallowing of two months duration. There was no loss of appetite, change of voice, or physical activity. Bowel habits were also normal. He has a negative history of cancers in the family or any thyroidal conditions. During the last month he noticed two new lumps on the right side of the neck as well. Upon examination, he was vitally stable and afebrile (BP: 113/71 Temperature: 37.3 Pulse: 81).
Neck examination showed a normal thyroid exam. However, there were three lumps on the right side of the neck, the biggest one was in the right posterior deep cervical region around $2 \mathrm{X} 1 \mathrm{~cm}$. The other one on the right submandibular group around $1 \mathrm{X} 1 \mathrm{~cm}$. The last lump noticed was over the right supraclavicular region and was the smallest around $0.5 \times 0.5 \mathrm{~cm}$. The rest of the examination was unremarkable. His laboratory tests showed Wbc: $13.9 \mathrm{Hb}$ 10.8. Remaining labs were all normal.

An ultrasound neck was done and revealed, multiple enlarged lymph nodes in the right supraclavicular and deep cervical groups. The largest measures $19 \times 10 \mathrm{~mm}$ and some of the nodes showed distorted hilum. Another enlarged lymph node noted in the left submandibular group measures $15 \times 8 \mathrm{~mm}$. Both thyroid lobes and isthmus were normal except for a nodule in the right lobe, which has the following criteria: R1 seen at the lower part of the lobe measures about (10x8x9.5mm) with mild surrounding vascularity, according to the ACR TI RADS its of TR5 which needs FNAC. ACR TI RADS CLASSIFICATION: it is mixed cystic and solid, hypoechoic, taller than wide, smooth, and punctate echogenic foci, which is highly suspicious for malignancy. An ultrasound guided fine needle aspiration of the thyroid nodule and the lymph nodes was done. The thyroid nodule showed a well-differentiated papillary thyroid cancer, and the lymph nodes showed a highgrade malignant tumor that needs a complete lymph node 


\section{Global Journal of Otolaryngology}

biopsy. Flow cytometry of the sample showed predominantly T-lymphocytes.

A pan CT was done, revealed multiple enlarged right upper and lower deep cervical lymph nodes in the neck, they are well defined, and homogenous, the largest measures about $19 \times 11 \mathrm{~mm}$, few other small left upper and lower deep cervical and bilateral supraclavicular lymph nodes noted. The largest one measures about $10 \times 6 \mathrm{~mm}$, also showed normal CT features of the nasopharynx, oropharynx, larynx, supraglottis, hypopharynx, vocal cords, pterygo-palatine, and pterygo-maxillary fossae, parapharyngeal and retropharyngeal spaces, parotid and submandibular glands, the rest of the paranasal sinuses were unremarkable.

CT chest showed, few small tissue nodules noted at the lateral segment of the right lower lobe and lateral segment of middle lobe of the lung, the largest one measures about $2 \times 2 \mathrm{~mm}$, with no dense calcifications inside. Other CT findings showed bilateral axillary lymph nodes, the largest measures about $10 \times 9 \mathrm{~mm}$. The course and caliber of the trachea and esophagus were normal, with no mediastinal and hilar lymphadenopathy. CT abdomen and pelvis showed right inguinal lymph nodes, the largest one about $11 \times 7 \mathrm{~mm}$. During his hospital stay, a multiple disciplinary team was engaged including the oncology. And with proper counseling of the patient, the decision was to go for right hemithyroidectomy and lymph node excisional biopsy. Patient underwent the surgery and was in good post-operative condition with no intra or postoperative complications. Samples sent for histopathology and came as multifocal papillary microcarcinoma of the thyroid and Hodgkin lymphoma (nodular sclerosis) from the enlarged lymph nodes (Figures 1-10).

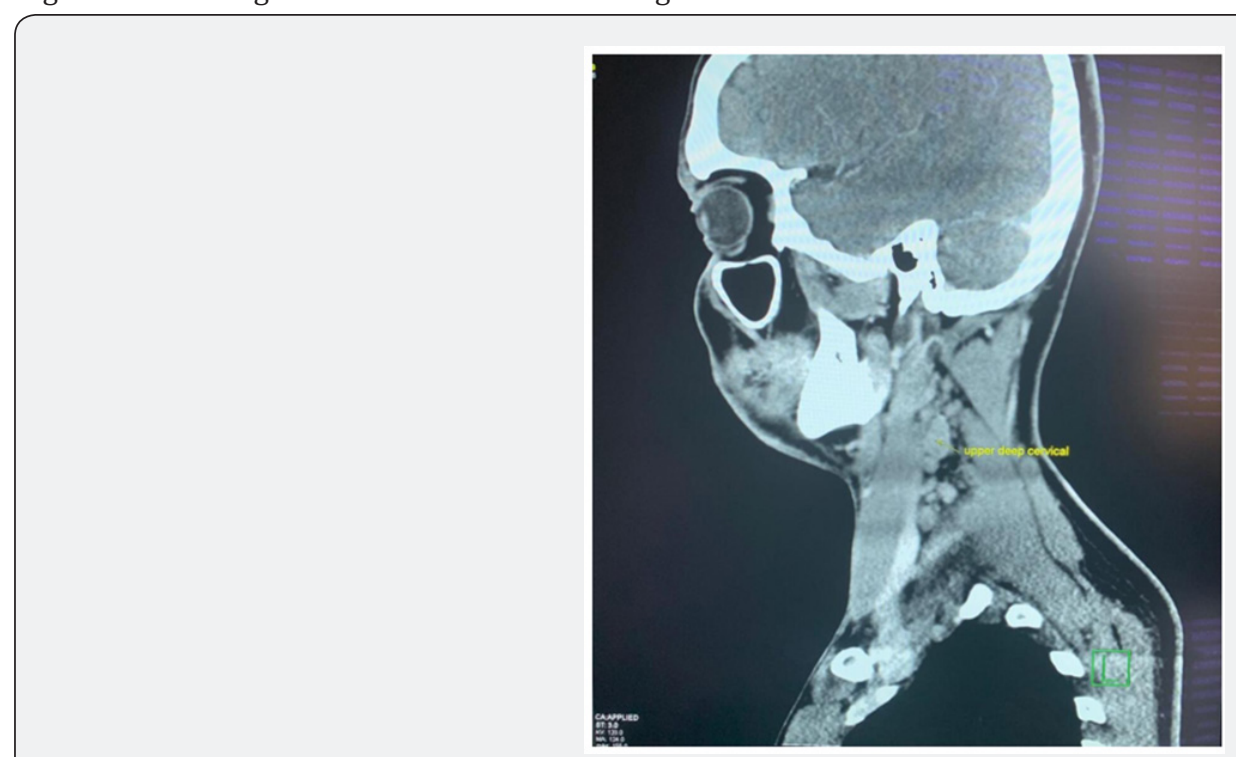

Figure 1: CT scan of the neck sagittal view yellow arrow showing right upper deep cervical LNs.

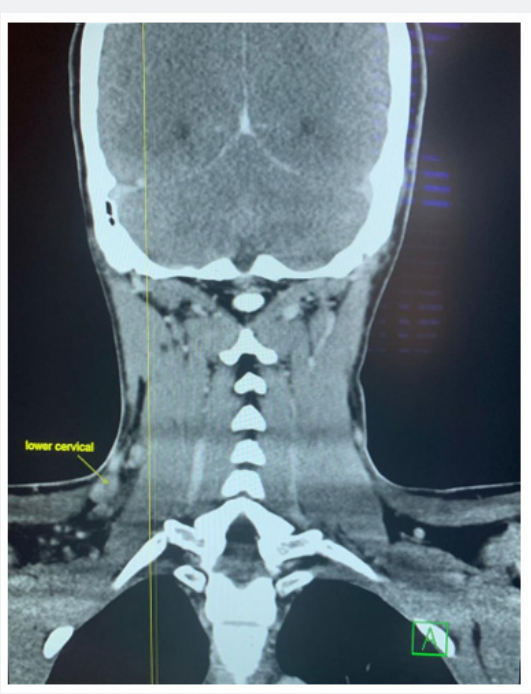

Figure 2: CT scan of the neck coronal view, yellow arrow showing right lower cervical LNs. 


\section{Global Journal of Otolaryngology}

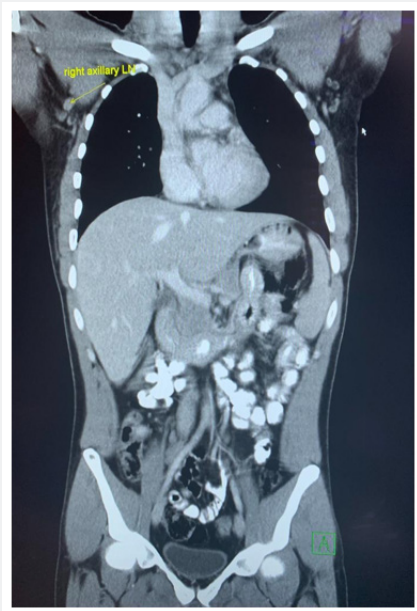

Figure 3: CT of chest and abdomen, yellow arrow showing right axillary LNs.

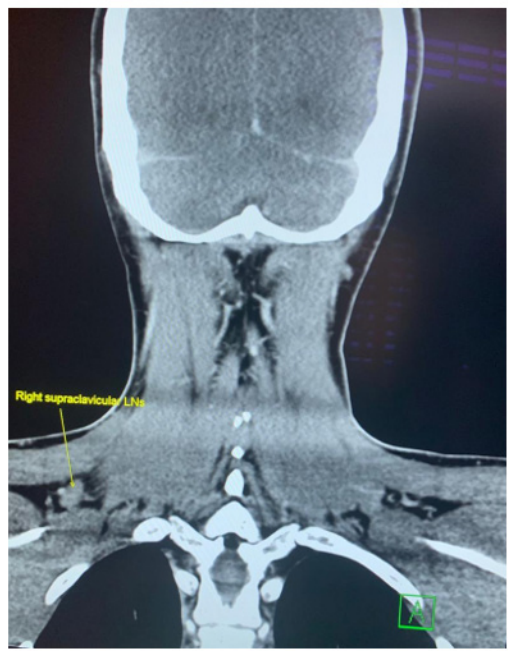

Figure 4: CT scan of the neck, yellow arrow showing right supraclavicular LNs.

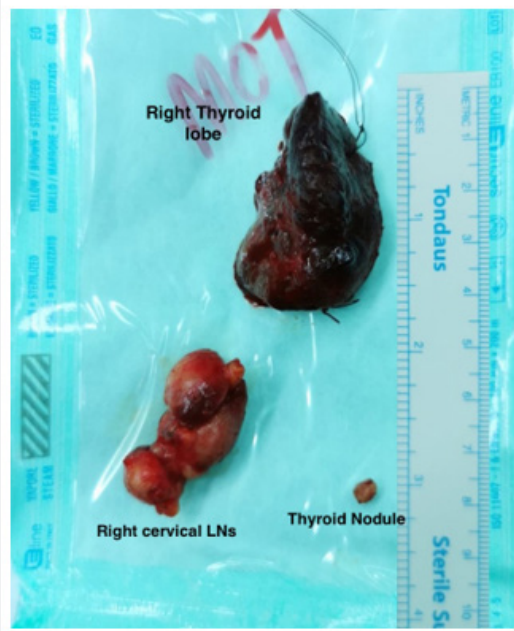

Figure 5: Gross excised samples of the right thyroid lobe, thyroid nodule, and the right cervical LNs. 


\section{Global Journal of Otolaryngology}

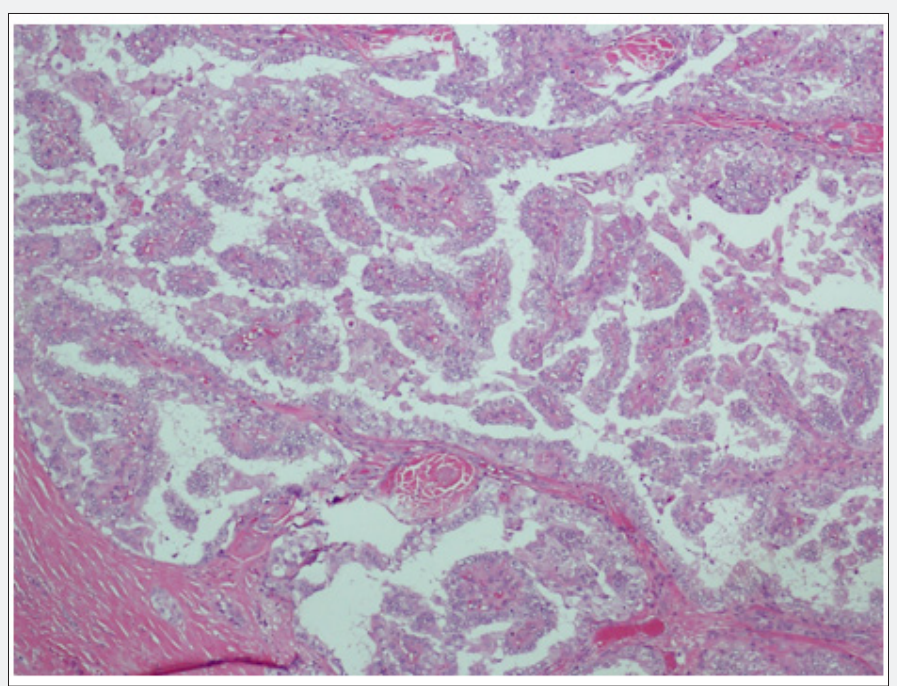

Figure 6: Papillary thyroid carcinoma. Papillae with ground glass (Orphan Annie eye ) nuclei. (Hematoxylin and Eosin stain, x100).

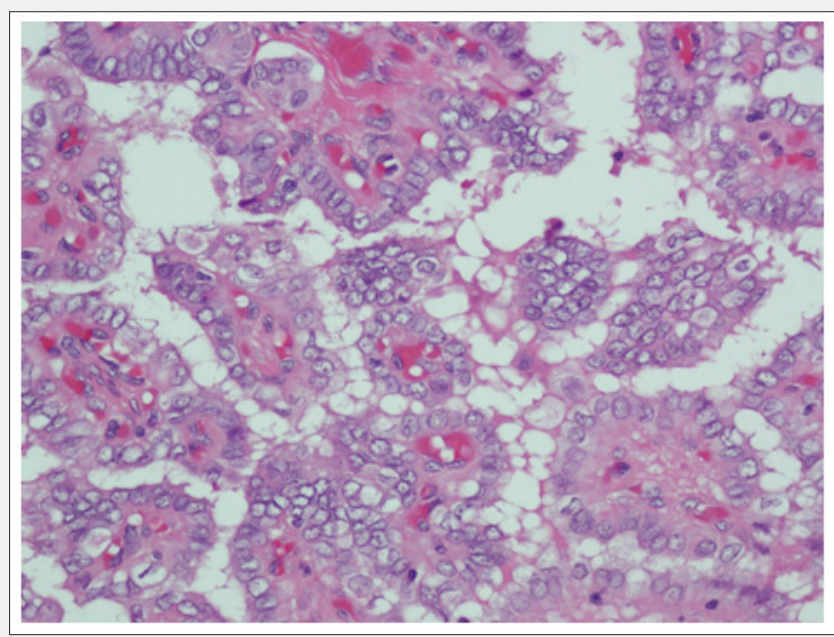

Figure 7: Papillary thyroid carcinoma. Tumour cells with nuclear enlargement, elongation and overlapping, having optically clear chromatin, irregular nuclear membranes, and nuclear grooves. (Hematoxylin and Eosin stain, x400).

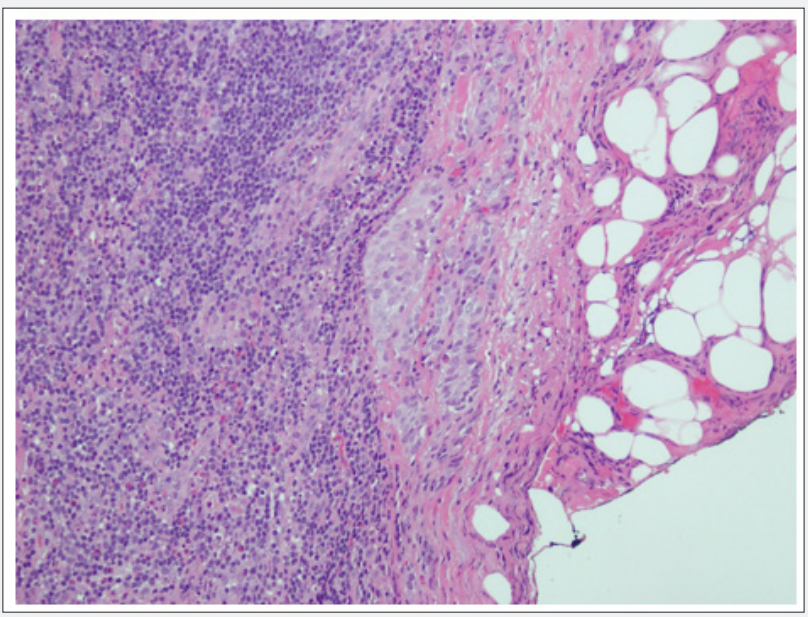

Figure 8: Lymph node with metastatic papillary thyroid carcinoma. (Hematoxylin and Eosin stain, x200) 


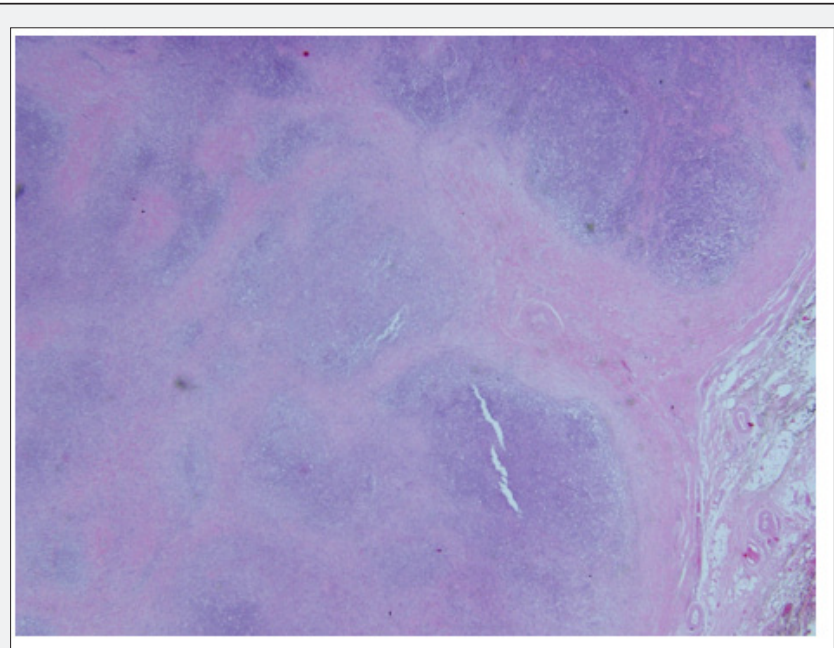

Figure 9: Lymph node with Classic Hodgkin lymphoma, Nodular sclerosis subtype. Broad bands of collagen dissect the lymph node into nodules. (Hematoxylin and Eosin stain, x20).

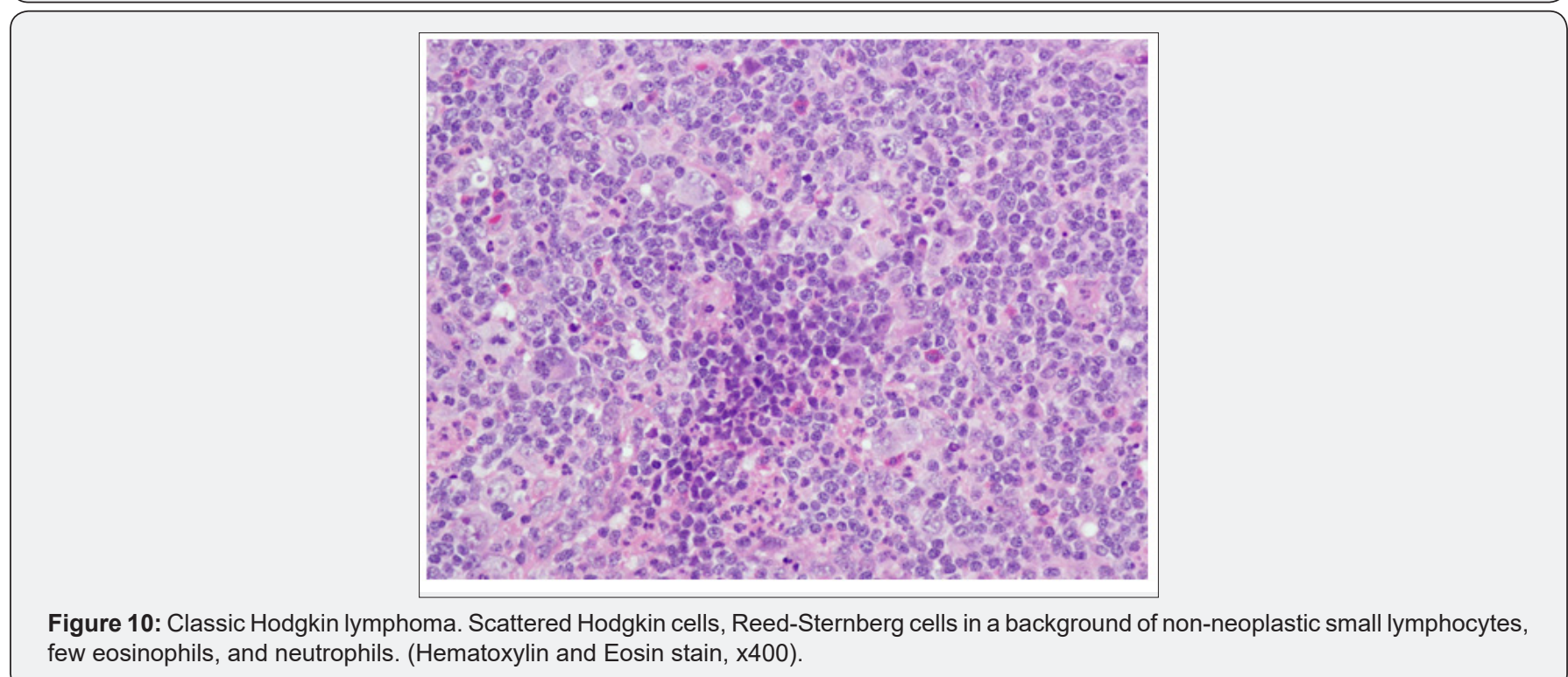

\section{Discussion}

Papillary thyroid cancer is the most common thyroid malignancy. Yet it has the best prognosis. The combination of both papillary thyroid cancer and hematological malignancies is considered extremely rare with an incident of $7 \%$. The incidence of papillary cancer has raised reaching 7.7 per 100.000. Most are detected in the early subclinical stage, concluding that the actual numbers did not increase it's just that the disease is being detected earlier [3]. When the second primary malignancy is detected in less than 6 months from the index tumor the term synchronous is implied. The clinical presentation of our patient of weight loss and night sweats in addition to the lateral neck swelling gave us a picture suggestive of lymphoma. However, with further investigations we were astonished that he had a concomitant papillary thyroid cancer [1]. Tumors especially thyroidal are initially diagnosed with FNAC, however the effectiveness on its own is controversial especially that its sensitivity is $55-86 \%$ [4].
Our case was further confirmed by the histopathology results proving different pathologies.

"Warren and Gates criteria for diagnosis of multiple primary malignancies include the following:

a. Each of the tumors must be malignancy confirmed by histology,

b. each must be geographically separate and distinct; the lesions should be separated by normal mucosa, and

c. probability of one being the metastasis of the other must be excluded" [5].

There is no clear link between Hodgkin's lymphoma and thyroid papillary cancer regarding risk factors whether genetically or environmental. However, a genetic mutation is always a possibility, but it has not yet revealed. On the other hand, the BRAF mutation has been found in papillary thyroid cancer and variable 
non-Hodgkin's lymphomas [6]. Literature suggested that breast carcinomas are the most diagnosed synchronous malignancies. Yet in metachronous malignancies, breast carcinoma and gynecological cancers were the commonest [1].

Risk factors that could possibly be associated with synchronous malignancies are constant carcinogen exposure from the surroundings, ozone exhaustion through years and ionizing radiation consequences, the need of organ transplantation, the use of hormonal manipulation for certain types of cancers, also target therapies included, genetic manipulation, and immunomodulators that modifies the functioning of the immune system [1]. In addition, radiotherapy might also be a risk factor. Certain studies showed the association of thyroid papillary carcinoma with lymphoma, mainly Hodgkin's lymphoma treated with radiotherapy. On the other hand, less than 10 cases of papillary thyroid cancer and Hodgkin's lymphoma did not receive radiotherapy [6].

After multi-disciplinary team consultation done including oncology, final decision was to do right hemithyroidectomy, since the size of the thyroid nodule was small, also lymph node excisional biopsy was done. Once the histopathology result of the lymph node biopsy showed Hodgkin lymphoma, chemotherapy was received according to the guidelines and protocol. According to our review of literature, some studies reported that subtotal thyroidectomy followed by radioiodine use without total thyroidectomy and lymphadenectomy would be an alternative option [7]. Georgi et al and colleagues [3], reported a similar case with different management, they first started with chemotherapy of lymphoma and then went for a total thyroidectomy. Up to this time there's no exact sequence of treatment of synchronous papillary thyroid cancer and Hodgkin lymphoma, that's due to its rarity and the scanty literature available. The presence of a second malignancy, require an instant and aggressive workup to prove there is two different pathologies, for early detection and management and for better outcomes and prognosis. The prognosis of such cases is very variable and mainly determined by the outcome of the most aggressive tumor [8].

\section{Conclusion}

This case delineates the extreme rarity of having synchronous incidence of papillary thyroid cancer and Hodgkin's lymphoma, in addition to the complexity of clinical management due to the limited literature available. It is highly important to perform thorough investigations to confirm the synchronous occurrence of two pathologies. As each pathology requires individual management for better patient outcome.

\section{References}

1. Panduranga G, Ali S T M, Thota N K (2019) A case report of synchronous primary malignancy: Papillary thyroid carcinoma and non-Hodgkin's lymphoma. Ind J Case Rep 5(1): 13-15.

2. Su S, Liu R, Hu Y, Gong G, Zhu W, et al. (2019) Neck Lumps with Enlarged Lymph Nodes: Papillary Thyroid Cancer and Hodgkin Lymphoma. Ann Case Rep ACRT-211.

3. Popivanov GI, Bochev P, Hristoskova R, Ventsislav M Mutafchiyski, Mihail Tabakov, et al. (2018) Synchronous papillary thyroid cancer and non-Hodgkin lymphoma: Case report. Medicine (Baltimore) 97(6): e9831.

4. Vicky Cheng, Jennifer Brainard, Christian Nasr (2012) Co-Occurrence of Papillary Thyroid Carcinoma and Primary Lymphoma of the Thyroid in a Patient with Long-Standing Hashimoto's Thyroiditis. Thyroid 22(6): 647-650.

5. Warren S, Gates O (1932) Multiple primary malignant tumors. A survey of the literature and a statistical study. Am J Cancer 16: 1358-1362.

6. Ahlem B, Nozha M, Marwa BN, Moncef M (2020) Concomitant of hodgkin lymphoma and papillary thyroid carcinoma. Otorhinolaryngol Head Neck Surg 5 .

7. Rizkallah JJ, Jambart SS, Maalouli GD (2014) Synchronous diagnosis of a hodgkin lymphoma and a papillary carcinoma of the thyroid. Case Rep Int Med 1: 235-237.

8. Al Gahmi A, Alhuthali M, Alrehaili M, Baltow B, Tashkandi E (2021) Unusual Synchronous Association of Solid Tumors with Hematological Malignancies in Multiple Primary Cancers: Case Series and Literature Review. Case Rep Oncol 14: 352-364. 
(C) Commons Attribution 4.0 License

(CC) DY 10.19080/GJO.2021.24.556143

\section{Your next submission with Juniper Publishers} will reach you the below assets

- Quality Editorial service

- Swift Peer Review

- Reprints availability

- E-prints Service

- Manuscript Podcast for convenient understanding

- Global attainment for your research

- Manuscript accessibility in different formats ( Pdf, E-pub, Full Text, Audio)

- Unceasing customer service

Track the below URL for one-step submission https://juniperpublishers.com/online-submission.php 